\title{
Global attractivity of a two-species competitive system with nonlinear inter-inhibition terms
}

\section{Baoguo Chen}

Research Center for Science Technology and Society, Fuzhou University of International Studies and Trade Fuzhou, Fujian, 350202, P. R. China.

\section{Abstract}

Sufficient conditions are obtained for the global attractivity of the positive equilibrium and boundary equilibria of the following two-species competitive system with nonlinear inter-inhibition terms

$$
\begin{aligned}
& \frac{d y_{1}(t)}{d t}=y_{1}(t)\left[r_{1}-a_{1} y_{1}-\frac{b_{1} y_{2}}{1+y_{2}}\right], \\
& \frac{d y_{2}(t)}{d t}=y_{2}(t)\left[r_{2}-a_{2} y_{2}-\frac{b_{2} y_{1}}{1+y_{1}}\right],
\end{aligned}
$$

where $r_{i}, a_{i}, b_{i}, i=1,2$ are all positive constants. Our result shows that conditions which ensure the permanence of the system are almost enough to ensure the global stability of the system. The results not only improve but also complement the main results of Wang et al. [Q. L. Wang, Z. J. Liu, Z. X. Li, R. A. Cheke, Int. J. Biomath., 7 (2014), 18 pages]. (c)2016 All rights reserved.

Keywords: Competition, nonlinear inter-inhibition terms, global attractivity.

$2010 M S C:$ 34C25, 92D25, 34D20, 34D40.

\section{Introduction}

The aim of this paper is to investigate the global dynamic behaviors of the following two-species competitive system with nonlinear inter-inhibition terms

Email address: chenbaoguo2016@163.com (Baoguo Chen) 


$$
\begin{aligned}
& \frac{d y_{1}(t)}{d t}=y_{1}(t)\left[r_{1}-a_{1} y_{1}-\frac{b_{1} y_{2}}{1+y_{2}}\right], \\
& \frac{d y_{2}(t)}{d t}=y_{2}(t)\left[r_{2}-a_{2} y_{2}-\frac{b_{2} y_{1}}{1+y_{1}}\right],
\end{aligned}
$$

where $r_{i}, a_{i}, b_{i}, i=1,2$ are all positive constants.

Recently, Wang et al. 23] studied the dynamic behaviors of the following two-species competitive system with nonlinear inter-inhibition terms

$$
\begin{aligned}
& \frac{d y_{1}(t)}{d t}=y_{1}(t)\left[r_{1}(t)-a_{1}(t) y_{1}-\frac{b_{1}(t) y_{2}}{1+y_{2}}\right], \\
& \frac{d y_{2}(t)}{d t}=y_{2}(t)\left[r_{2}(t)-a_{2}(t) y_{2}-\frac{b_{2}(t) y_{1}}{1+y_{1}}\right],
\end{aligned}
$$

where $y_{i}(i=1,2)$ are the population densities of two competing species at time $t ; r_{i}(t)(i=1,2)$ are the intrinsic growth rates of species $i ; a_{i}(i=1,2)$ are the rates of intraspecific competition of the first and second species, respectively; and $b_{i}(t)(i=1,2)$ are the rates of intraspecific competition of the first and second species, respectively. For more background of system (1.1), we refer the reader to [11, 19, 21 23, 28] and the references cited therein. For an almost periodic function $f(t)$, set $f^{L}=\inf _{t \in R} f(t), f^{U}=\sup _{t \in R} f(t)$. Under the assumption $r_{i}(t), a_{i}(t)$ and $b_{i}(t), i=1,2$ are all positive almost periodic functions defined on $R^{+}=[0,+\infty)$. The authors investigated the existence and global asymptotic stability of positive almost periodic solutions of the system $(1.2)$, they obtained the following results:

Theorem 1.1. Any positive solution $\left(y_{1}(t), y_{2}(t)\right)$ of system 1.2 satisfies

$$
\limsup _{t \rightarrow+\infty} y_{i}(t) \leq M_{i}=\frac{r_{i}^{U}}{a_{i}^{L}}, i=1,2 .
$$

Theorem 1.2. If the following assumptions

$$
r_{1}^{L}>\left(b_{1}^{U}-r_{1}^{L}\right) M_{2}, r_{2}^{L}>\left(b_{2}^{U}-r_{2}^{L}\right) M_{1}
$$

are satisfied, then any positive solution $\left(y_{1}(t), y_{2}(t)\right)$ of system 1.2 satisfies

$$
\liminf _{t \rightarrow+\infty} y_{i}(t) \geq m_{i}=\frac{r_{i}^{L}+\left(r_{i}^{L}-b_{i}^{U}\right) M_{j}}{a_{i}^{U}\left(1+M_{j}\right)}, i, j=1,2 ; i \neq j .
$$

Theorem 1.3. If the almost periodic parameters $r_{i}(t), a_{i}(t), b_{i}(t)(i=1,2)$ of system (1.2) satisfies (1.3) and

$$
a_{1}^{L}-\frac{b_{2}^{U}}{\left(1+m_{1}\right)^{2}}>0, a_{2}^{L}-\frac{b_{1}^{U}}{\left(1+m_{2}\right)^{2}}>0,
$$

then system (1.2) has a globally asymptotically stable positive almost periodic solution.

It brings to our attention that the authors had investigated the stability property of the system (1.2) by constructing some suitable Lyapunov function, generally speaking, the conditions obtained by using Lyapunov function are very complicated, the additional condition, to some extent, is necessary. But for the system itself, this condition may not be necessary. Also, the authors in [23] did not investigated the extinction property of the system (1.2), which is one of the most important topics in the study of population dynamics (see [2 [5, 7, ㅇ, 12, 13, 18, 20] and the references therein). Above 
analysis motivated us to revisit the autonomous case of system 1.2 , i.e., system (1.1).

From the point of view of biology, in the sequel, we shall consider (1.1) together with the initial conditions

$$
y_{i}(0)>0, \quad i=1,2 .
$$

Obviously, system(1.1) has a unique solution $\left(y_{1}(t), y_{2}(t)\right)$ satisfying the initial condition (1.6). We easily prove $y_{i}(t)>0$ for all $i=1,2$ in maximal interval of existence of the solution.

The aim of this paper is, by further developing the analysis technique of [1, 6, 9, 10, 14, 17, 24[27, 29] and using the differential inequality theory, to investigate the global dynamic behaviors of the system (1.1). More precisely, we will prove the following results.

Theorem 1.4. Assume that the following inequalities

$$
r_{1}\left(a_{2}+r_{2}\right)>b_{1} r_{2}, \quad r_{2}\left(a_{1}+r_{1}\right)>b_{2} r_{1}
$$

hold, and assume further that one of the following conditions holds,

(A)

$$
a_{2}-b_{2}+r_{2} \neq 0
$$

(B)

$$
a_{2}-b_{2}+r_{2}=0, a_{1} r_{2}-a_{2} r_{1}>0
$$

then system (1.1) admits a unique positive equilibrium $\left(y_{1}^{*}, y_{2}^{*}\right)$, which is globally attractive, that is, for any positive solution $\left(y_{1}(t), y_{2}(t)\right)$ of system (1.1) with the initial condition (1.6), one has

$$
\lim _{t \rightarrow+\infty} y_{i}(t)=y_{i}^{*}, i=1,2 .
$$

Remark 1.5. For system (1.1), the first inequality $r_{1}^{L}>\left(b_{1}^{U}-r_{1}^{L}\right) M_{2}$ in (1.3) is equivalent to $r_{1}>\left(b_{1}-\right.$ $\left.r_{1}\right) \frac{r_{2}}{a_{2}}$, or $r_{1}\left(a_{2}+r_{2}\right)>b_{1} r_{2}$. Similarly, the second inequality in (1.3) is equivalent to $r_{2}\left(a_{1}+r_{1}\right)>b_{2} r_{1}$. Therefore, Theorem 1.4 shows that for the autonomous case of system (1.2), the conditions which ensure the permanence of the system are almost enough to ensure the global stability of the system, only the degenerate case $a_{2}-b_{2}+r_{2}=0$ needs further consideration.

As a direct corollary of Theorem 1.4 , we have,

Corollary 1.6. Assume that the following inequalities

$$
r_{1}>b_{1}, r_{2}>b_{2}
$$

hold, then system (1.1) admits a unique positive equilibrium $\left(y_{1}^{*}, y_{2}^{*}\right)$, which is globally attractive, that is, for any positive solution $\left(y_{1}(t), y_{2}(t)\right)$ of system (1.1) with the initial condition (1.6), one has:

$$
\lim _{t \rightarrow+\infty} y_{i}(t)=y_{i}^{*}, i=1,2 .
$$

Theorem 1.7. Assume that the following inequalities

$$
r_{1}\left(a_{2}+r_{2}\right)>b_{1} r_{2}, r_{2}-\frac{b_{2} m_{1}}{1+m_{1}}<0
$$

hold, where 


$$
m_{1}=\frac{r_{1}-\frac{b_{1} M_{2}}{1+M_{2}}}{a_{1}}, \quad M_{2}=\frac{r_{2}}{a_{2}}
$$

then

$$
\lim _{t \rightarrow+\infty} y_{1}(t)=\frac{r_{1}}{a_{1}}, \lim _{t \rightarrow+\infty} y_{2}(t)=0 .
$$

Remark 1.8. Condition 1.10 is equivalent to

$$
\begin{gathered}
r_{1}\left(a_{2}+r_{2}\right)>b_{1} r_{2}, \\
\left(a_{1}-b_{1}+r_{1}\right) r_{2}^{2}+\left(a_{1} a_{2}+a_{2} r_{1}+b_{1} b_{2}-b_{2} r_{1}\right) r_{2}-a_{2} b_{2} r_{1}<0 .
\end{gathered}
$$

One could easily see that if $r_{1}$ is large enough and $r_{2}$ is small enough, then (1.11) always holds, and the second species will be driven to extinction. That is, for system (1.1), the intrinsic growth rate plays important role on the persistent and extinction property of the species. Large intrinsic growth rate will improve the chance of the survival of the species.

Theorem 1.9. Assume that the following inequalities

$$
r_{1}-\frac{b_{1} m_{2}}{1+m_{2}}<0, \quad r_{2}\left(a_{1}+r_{1}\right)>b_{2} r_{1}
$$

hold, where

$$
m_{2}=\frac{r_{2}-\frac{b_{2} M_{1}}{1+M_{1}}}{a_{2}}, \quad M_{1}=\frac{r_{1}}{a_{1}}
$$

then

$$
\lim _{t \rightarrow+\infty} y_{1}(t)=0, \lim _{t \rightarrow+\infty} y_{2}(t)=\frac{r_{2}}{a_{2}} .
$$

Remark 1.10. Condition 1.12 is equivalents to

$$
\begin{gathered}
\left(a_{2}-b_{2}+r_{2}\right) r_{1}^{2}+\left(a_{1} a_{2}+a_{1} r_{2}+b_{1} b_{2}-b_{1} r_{2}\right) r_{1}-a_{1} b_{2} r_{2}<0, \\
r_{2}\left(a_{1}+r_{1}\right)>b_{2} r_{1} .
\end{gathered}
$$

One can easily see that if $r_{2}$ is large enough and $r_{1}$ is small enough, then (1.13) always holds, and the first species will be driven to extinction.

The rest of the paper is arranged as follows: We will introduce some useful lemmas in the next section, and then prove the main results in Section 3. Some numeric simulations are carried out in Section 4, and we end this paper by a brief discussion.

\section{Lemmas}

Now let us state several lemmas which will be useful in the proving of main results.

Lemma 2.1. In addition to (1.7), further assume that (1.8) or (1.9) holds, then system (1.1) admits a unique positive equilibrium $\left(y_{1}^{*}, y_{2}^{*}\right)$.

Proof. The positive equilibrium of system (1.1) satisfies the following equation

$$
\begin{aligned}
& r_{1}-a_{1} y_{1}-\frac{b_{1} y_{2}}{1+y_{2}}=0, \\
& r_{2}-a_{2} y_{2}-\frac{b_{2} y_{1}}{1+y_{1}}=0 .
\end{aligned}
$$


Since we focus on the positive solution of the system 1.1 , it implies that we only need to consider the case $y_{1}>0, y_{2}>0$. Hence, to ensure the first equality holds, $y_{1}$ should be lied in the interval $\left(0, \frac{r_{1}}{a_{1}}\right)$. Similarly, to ensure the second equality holds, $y_{2}$ should be lied in the interval $\left(0, \frac{r_{2}}{a_{2}}\right)$. In th following we will investigate the positive equilibrium of system 1.1$)$ on the rectangle $\left(0, \frac{r_{1}}{a_{1}}\right) \times\left(0, \frac{r_{2}}{a_{2}}\right)$.

From the second equation of system (2.1) one can obtain

$$
y_{2}=-\frac{b_{2} y_{1}-r_{2} y_{1}-r_{2}}{a_{2}\left(1+y_{1}\right)}
$$

Substituting (2.2) into the first equation of 2.1) leads to

$$
A_{1} y_{1}^{2}+A_{2} y_{1}+A_{3}=0
$$

where

$$
\begin{aligned}
& A_{1}=a_{1} a_{2}-a_{1} b_{2}+a_{1} r_{2}, \\
& A_{2}=a_{1} a_{2}+a_{1} r_{2}-a_{2} r_{1}-b_{1} b_{2}+b_{1} r_{2}+b_{2} r_{1}-r_{1} r_{2}, \\
& A_{3}=-a_{2} r_{1}+b_{1} r_{2}-r_{1} r_{2} .
\end{aligned}
$$

Now let us consider the function

$$
F\left(y_{1}\right)=A_{1} y_{1}^{2}+A_{2} y_{1}+A_{3}
$$

Since

$$
F(0)=A_{3}<0
$$

and

$$
F\left(\frac{r_{1}}{a_{1}}\right)=\frac{b_{1}\left(a_{1} r_{2}-b_{2} r_{1}+r_{1} r_{2}\right)}{a_{1}}>0,
$$

which means that $F\left(y_{1}\right)=0$ has at least one solution on the interval $\left(0, \frac{r_{1}}{a_{1}}\right)$.

Now let us show that under the assumption of the lemma, $F\left(y_{1}\right)=0$ has at most one positive solution on the interval $\left(0, \frac{r_{1}}{a_{1}}\right)$.

(1) Assume that $a_{2}-b_{2}+r_{2}>0$, in this case, $F(+\infty)=F(-\infty)=+\infty$, since $F(0)<0$, it follows that $F\left(y_{1}\right)$ has at least one solution on the interval $(-\infty, 0)$ and $(0,+\infty)$, respectively. Since $F\left(y_{1}\right)=0$ has at most two solutions, it follows that $F\left(y_{1}\right)=0$ has at most one solution on the interval $\left(0, \frac{r_{1}}{a_{1}}\right)$;

(2) Assume that $a_{2}-b_{2}+r_{2}<0$, in this case, $F(+\infty)=-\infty$, since $F(0)<0, F\left(\frac{r_{1}}{a_{1}}\right)>0$, it follows that $F\left(y_{1}\right)$ has at least one solution on the interval $\left(0, \frac{r_{1}}{a_{1}}\right)$ and $\left(\frac{r_{1}}{a_{1}},+\infty\right)$, respectively. Since $F\left(y_{1}\right)=0$ has at most two solutions, it follows that $F\left(y_{1}\right)=0$ has at most one solution on the interval $\left(0, \frac{r_{1}}{a_{1}}\right)$;

(3) Assume that $a_{2}-b_{2}+r_{2}=0$, in this case, $F(0)<0$, also, from 1.9 one has $F\left(\frac{r_{1}}{a_{1}}\right)=$ $a_{1} b_{1} r_{2}-a_{2} b_{1} r_{1}>0$, since $F\left(y_{1}\right)$ is the linear function of $y_{1}$, it immediately follows that $F\left(y_{1}\right)=0$ has only one solution on the interval $\left(0, \frac{r_{1}}{a_{1}}\right)$.

Above analysis shows that under the assumption of Lemma 2.1, $F\left(y_{1}\right)=0$ has at most one positive solution on the interval $\left(0, \frac{r_{1}}{a_{1}}\right)$. Therefore, $F\left(y_{1}\right)=0$ has a unique positive solution on the interval $\left(0, \frac{r_{1}}{a_{1}}\right)$. Set this solution as $y_{1}^{*}$. Also, from 2.2 we have

$$
y_{2}^{\prime}\left(y_{1}\right)=-\frac{b_{2}}{a_{2}(1+x)^{2}}<0 \text {. }
$$


Since $y_{2}(0)=\frac{r_{2}}{a_{2}}$ and $y_{2}\left(\frac{r_{1}}{a_{1}}\right)=\frac{a_{1} r_{2}-b_{2} r_{1}+r_{1} r_{2}}{a_{2}\left(a_{1}+r_{1}\right)}>0$, it follows that $y_{2}\left(y_{1}^{*}\right)>0$. Set $y_{2}\left(y_{1}^{*}\right)=y_{2}^{*}$. Then system (1.1) admits a unique positive equilibrium $\left(y_{1}^{*}, y_{2}^{*}\right)$. This ends the proof of Lemma 2.1 .

As a direct corollary of Lemma 2.2 of [1] by Chen, we have,

Lemma 2.2. If $a>0, b>0$ and $\dot{x} \geq x(b-a x)$, when $t \geq 0$ and $x(0)>0$, we have

$$
\liminf _{t \rightarrow+\infty} x(t) \geq \frac{b}{a}
$$

If $a>0, b>0$ and $\dot{x} \leq x(b-a x)$, when $t \geq 0$ and $x(0)>0$, we have

$$
\limsup _{t \rightarrow+\infty} x(t) \leq \frac{b}{a}
$$

\section{Proof of the main results}

Now we are in the position to prove the main results of this paper.

Proof of Theorem 1.4. It follows from (1.7) that there exists an $\varepsilon>0$ small enough such that

$$
r_{1}>\frac{b_{1}\left(\frac{r_{2}}{a_{2}}+\varepsilon\right)}{1+\left(\frac{r_{2}}{a_{2}}+\varepsilon\right)}+a_{1} \varepsilon, \quad r_{2}>\frac{b_{2}\left(\frac{r_{1}}{a_{1}}+\varepsilon\right)}{1+\left(\frac{r_{1}}{a_{1}}+\varepsilon\right)}+a_{2} \varepsilon .
$$

Let $\left(y_{1}(t), y_{2}(t)\right)$ be any positive solution of system (1.1) with initial condition (1.6). From system (1.1) it follows that

$$
\frac{d y_{i}(t)}{d t} \leq y_{i}(t)\left[r_{i}-a_{i} y_{i}\right]
$$

Thus, as a direct corollary of Lemma 2.2, according to 3.1 , one has

$$
\limsup _{t \rightarrow+\infty} y_{i}(t) \leq \frac{r_{i}}{a_{i}}
$$

Hence, for small enough $\varepsilon>0$, it follows from that there exists a $T_{1}>0$ such that

$$
y_{i}(t)<\frac{r_{i}}{a_{i}}+\varepsilon \stackrel{\text { def }}{=} M_{i}^{(1)}, \quad i=1,2
$$

For $t>T_{1}$, it follows from the first equation of system 1.1 that

$$
\frac{d y_{1}(t)}{d t} \geq y_{1}(t)\left[r_{1}-a_{1} y_{1}-\frac{b_{1} M_{2}^{(1)}}{1+M_{2}^{(1)}}\right] .
$$

Thus, as a direct corollary of Lemma 2.2, according to 3.5$)$, one has

$$
\liminf _{t \rightarrow+\infty} y_{1}(t) \geq \frac{r_{1}-\frac{b_{1} M_{2}^{(1)}}{1+M_{2}^{(1)}}}{a_{1}} .
$$


Hence, for small enough $\varepsilon>0$, satisfying (3.1), it follows from (3.1) and (3.6) that there exists a $T_{2}^{\prime}>0$ such that

$$
y_{1}(t)>\frac{r_{1}-\frac{b_{1} M_{2}^{(1)}}{1+M_{2}^{(1)}}}{a_{1}}-\varepsilon \stackrel{\text { def }}{=} m_{1}^{(1)} \text { for } t>T_{2}^{\prime} .
$$

Similarly, for above $\varepsilon>0$, it follows from the second equation of system (1.1) that there exists a $T_{2}>T_{2}^{\prime}$ such that

$$
y_{2}(t)>\frac{r_{2}-\frac{b_{2} M_{1}^{(1)}}{1+M_{1}^{(1)}}}{a_{2}}-\varepsilon \stackrel{\text { def }}{=} m_{2}^{(1)} \text { for } t>T_{2} .
$$

For $t>T_{2}$, it follows from the first equation of system (1.1) that

$$
\frac{d y_{1}(t)}{d t} \leq y_{1}(t)\left[r_{1}-a_{1} y_{1}-\frac{b_{1} m_{2}^{(1)}}{1+m_{2}^{(1)}}\right] .
$$

Thus, as a direct corollary of Lemma 2.2 , according to $(3.8)$, one has

$$
\limsup _{t \rightarrow+\infty} y_{1}(t) \leq \frac{r_{1}-\frac{b_{1} m_{2}^{(1)}}{1+m_{2}^{(1)}}}{a_{1}} .
$$

Hence, for $\varepsilon>0$ satisfying (3.1), it follows from $(3.9)$ that there exists a $T_{3}^{\prime}>0$ such that

$$
y_{1}(t)<\frac{r_{1}-\frac{b_{1} m_{2}^{(1)}}{1+m_{2}^{(1)}}}{a_{1}}+\frac{\varepsilon}{2} \stackrel{\text { def }}{=} M_{1}^{(2)} \text { for } t>T_{3}^{\prime} .
$$

Similarly, for above $\varepsilon>0$, it follows from the second equation of system (1.1) that there exists a $T_{3}>T_{3}^{\prime}$ such that

$$
y_{2}(t)<\frac{r_{2}-\frac{b_{2} m_{1}^{(1)}}{1+m_{1}^{(1)}}}{a_{2}}+\frac{\varepsilon}{2} \stackrel{\text { def }}{=} M_{2}^{(2)} \text { for } t>T_{3} .
$$

For $t>T_{3}$, it follows from the first equation of system (1.1) that

$$
\frac{d y_{1}(t)}{d t} \geq y_{1}(t)\left[r_{1}-a_{1} y_{1}-\frac{b_{1} M_{2}^{(2)}}{1+M_{2}^{(2)}}\right] .
$$

Thus, as a direct corollary of Lemma 2.2 , according to $(3.8)$, one has

$$
\liminf _{t \rightarrow+\infty} y_{1}(t) \geq \frac{r_{1}-\frac{b_{1} M_{2}^{(2)}}{1+M_{2}^{(2)}}}{a_{1}} .
$$

Hence, for $\varepsilon>0$ satisfying (3.1), it follows from 3.10 that there exists a $T_{4}^{\prime}>0$ such that

$$
y_{1}(t)>\frac{r_{1}-\frac{b_{1} M_{2}^{(2)}}{1+M_{2}^{(2)}}}{a_{1}}-\frac{\varepsilon}{2} \stackrel{\text { def }}{=} m_{1}^{(2)} \text { for } t>T_{4}^{\prime} .
$$

Similarly, for above $\varepsilon>0$, it follows from the second equation of system (1.1) that there exists a 
$T_{4}>T_{4}^{\prime}$ such that

$$
y_{2}(t)>\frac{r_{2}-\frac{b_{2} M_{1}^{(2)}}{1+M_{1}^{(2)}}}{a_{2}}-\frac{\varepsilon}{2} \stackrel{\text { def }}{=} m_{2}^{(2)} \text { for } t>T_{4} .
$$

One can easily see that

$$
\begin{aligned}
M_{1}^{(2)}= & \frac{r_{1}-\frac{b_{1} m_{2}^{(1)}}{1+m_{2}^{(1)}}}{a_{1}}+\frac{\varepsilon}{2}<\frac{r_{1}}{a_{1}}+\varepsilon=M_{1}^{(1)}, \\
M_{2}^{(2)}= & \frac{r_{2}-\frac{b_{2} m_{1}^{(1)}}{1+m_{1}^{(1)}}}{a_{2}}+\frac{\varepsilon}{2}<\frac{r_{2}}{a_{2}}+\varepsilon=M_{2}^{(1)}, \\
m_{1}^{(2)}= & \frac{r_{1}-\frac{b_{1} M_{2}^{(2)}}{1+M_{2}^{(2)}}}{a_{1}}-\frac{\varepsilon}{2}>\frac{r_{1}-\frac{b_{1} M_{2}^{(1)}}{1+M_{2}^{(1)}}}{a_{1}}-\varepsilon=m_{1}^{(1)}, \\
m_{2}^{(2)}= & \frac{b_{2} M_{1}^{(2)}}{1+M_{1}^{(2)}}-\frac{b_{2}}{a_{2}}>\frac{r_{2}-\frac{b_{1}}{1+M_{1}^{(1)}}}{a_{2}}-\varepsilon=m_{2}^{(1)} .
\end{aligned}
$$

Repeating the above procedure, we get four sequences $M_{i}^{(n)}, m_{i}^{(n)}, i=1,2, n=1,2, \cdots$, such that for $n \geq 2$

$$
\begin{aligned}
M_{1}^{(n)}= & \frac{r_{1}-\frac{b_{1} m_{2}^{(n-1)}}{1+m_{2}^{(n-1)}}}{a_{1}}+\frac{\varepsilon}{n}, \quad M_{2}^{(n)}=\frac{r_{2}-\frac{b_{2} m_{1}^{(n-1)}}{1+m_{1}^{(n-1)}}}{a_{2}}+\frac{\varepsilon}{n}, \\
m_{1}^{(n)}= & \frac{r_{1}-\frac{b_{1} M_{2}^{(n)}}{1+M_{2}^{(n)}}}{a_{1}}-\frac{\varepsilon}{n}, \quad m_{2}^{(n)}=\frac{r_{2}-\frac{b_{2} M_{1}^{(n)}}{1+M_{1}^{(n)}}}{a_{2}}-\frac{\varepsilon}{n} .
\end{aligned}
$$

Obviously,

$$
m_{i}^{(n)}<N_{i}(t)<M_{i}^{(n)}, \text { for } t \geq T_{2 n}, \quad i=1,2 .
$$

We claim that sequences $M_{i}^{(n)}, i=1,2$ are non-increasing, and sequences $m_{i}^{(n)}, i=1,2$ are nondecreasing. To prove this claim, we will carry out by the induction. Firstly, from (3.11) we have

$$
M_{i}^{(2)}<M_{i}^{(1)}, \quad m_{i}^{(2)}>m_{i}^{(1)}, \quad i=1,2 .
$$

Let us assume now that our claim is true for $n$, that is,

$$
M_{i}^{(n)}<M_{i}^{(n-1)}, \quad m_{i}^{(n)}>m_{i}^{(n-1)}, \quad i=1,2 .
$$

Again from the strictly increasing of function of $g(x)=\frac{x}{1+x}$, we immediately obtain

$$
M_{1}^{(n+1)}=\frac{r_{1}-\frac{b_{1} m_{2}^{(n)}}{1+m_{2}^{(n)}}}{a_{1}}+\frac{\varepsilon}{n+1}<\frac{r_{1}-\frac{b_{1} m_{2}^{(n-1)}}{1+m_{2}^{(n-1)}}}{a_{1}}+\frac{\varepsilon}{n}=M_{1}^{(n)}
$$




$$
\begin{aligned}
M_{2}^{(n+1)}= & \frac{r_{2}-\frac{b_{2} m_{1}^{(n)}}{1+m_{1}^{(n)}}}{a_{2}}+\frac{\varepsilon}{n+1}<\frac{r_{2}-\frac{b_{2} m_{1}^{(n-1)}}{1+m_{1}^{(n-1)}}}{a_{2}}+\frac{\varepsilon}{n}=M_{2}^{(n)}, \\
m_{1}^{(n+1)}= & \frac{r_{1}-\frac{b_{1} M_{2}^{(n+1)}}{1+M_{2}^{(n+1)}}}{a_{1}}-\frac{\varepsilon}{n+1}>\frac{r_{1}-\frac{b_{1} M_{2}^{(n)}}{1+M_{2}^{(n)}}}{a_{1}}-\frac{\varepsilon}{n}=m_{1}^{(n)}, \\
m_{2}^{(n+1)}= & \frac{r_{2}-\frac{b_{2} M_{1}^{(n+1)}}{1+M_{1}^{(n+1)}}}{a_{2}}-\frac{\varepsilon}{n+1}>\frac{r_{2}-\frac{b_{2} M_{1}^{(n)}}{1+M_{1}^{(n)}}}{a_{2}}-\frac{\varepsilon}{n}=m_{2}^{(n)} .
\end{aligned}
$$

Therefore,

$$
\lim _{t \rightarrow+\infty} M_{i}^{(n)}=\bar{y}_{i}, \quad \lim _{t \rightarrow+\infty} m_{i}^{(n)}=\underline{y}_{i}, \quad i=1,2 .
$$

Letting $n \rightarrow+\infty$ in 3.12 , we obtain

$$
\begin{aligned}
& a_{1} \bar{y}_{1}=r_{1}-\frac{b_{1} \underline{y}_{2}}{1+\underline{y}_{2}}, \quad a_{2} \underline{y}_{2}=r_{2}-\frac{b_{2} \bar{y}_{1}}{1+\bar{y}_{1}}, \\
& a_{1} \underline{y}_{1}=r_{1}-\frac{b_{1} \bar{y}_{2}}{1+\bar{y}_{2}}, \quad a_{2} \bar{y}_{2}=r_{2}-\frac{b_{2} \underline{y}_{1}}{1+\underline{y}_{1}} .
\end{aligned}
$$

Equation (3.13) shows that $\left(\bar{y}_{1}, \underline{y}_{2}\right)$ and $\left(\underline{y}_{1}, \bar{y}_{2}\right)$ are solutions of 2.1). By Lemma 2.1, equation 2.1) has a unique positive solution $E^{*}\left(y_{1}^{*}, y_{2}^{*}\right)$. Hence, we conclude that

$$
\bar{y}_{i}=\underline{y}_{i}=N_{i}^{*}, \quad i=1,2,
$$

that is,

$$
\lim _{t \rightarrow+\infty} y_{i}(t)=y_{i}^{*} \quad i=1,2 .
$$

Thus, the unique interior equilibrium $E^{*}\left(y_{1}^{*}, y_{2}^{*}\right)$ is globally attractive. This completes the proof of Theorem 1.4.

Proof of Theorem 1.7. Condition

$$
r_{1}\left(a_{2}+r_{2}\right)>b_{1} r_{2}, r_{2}-\frac{b_{2} m_{1}}{1+m_{1}}<0
$$

implies that there exists a small enough $\varepsilon$ such that

$$
r_{1}>\frac{b_{1}\left(\frac{r_{2}}{a_{2}}+\varepsilon\right)}{1+\left(\frac{r_{2}}{a_{2}}+\varepsilon\right)}+a_{1} \varepsilon, \quad r_{2}-\frac{b_{2} m_{1}^{\varepsilon}}{1+m_{1}^{\varepsilon}}<0
$$

holds, where

$$
m_{1}^{\varepsilon}=\frac{r_{1}-\frac{b_{1} M_{2}^{\varepsilon}}{1+M_{2}^{\varepsilon}}}{a_{1}}, \quad M_{2}^{\varepsilon}=\frac{r_{2}}{a_{2}}+\varepsilon .
$$

Similar to the analysis of (3.2)-(3.7), there exists a $T>0$ such that

$$
y_{i}(t)<\frac{r_{i}}{a_{i}}+\varepsilon=M_{i}^{\varepsilon}, \quad i=1,2
$$


and

$$
y_{1}(t)>\frac{r_{1}-\frac{b_{1} M_{2}^{\varepsilon}}{1+M_{2}^{\varepsilon}}}{a_{1}}-\varepsilon \stackrel{\text { def }}{=} m_{1}^{\varepsilon} .
$$

From the second equation of system (1.1) and (3.14), we have

$$
\frac{d y_{2}(t)}{d t}<y_{2}(t)\left[r_{2}-\frac{b_{2} m_{1}^{\varepsilon}}{1+m_{1}^{\varepsilon}}\right]
$$

Hence,

$$
y_{2}(t)=y_{2}(T) \exp \left\{\int_{T}^{t}\left[r_{2}-\frac{b_{2} m_{1}^{\varepsilon}}{1+m_{1}^{\varepsilon}}\right] d t\right\} \rightarrow 0 \text { as } t \rightarrow+\infty
$$

For small enough $\varepsilon_{1}>0$, it follows from 3.15 that there exists a $T_{1}>T$ such that

$$
0<y_{2}(t)<\varepsilon_{1} \text { for all } t \geq T_{1} \text {. }
$$

It follows from $(3.16)$ and the first equation of $(1.1)$ that

$$
\frac{d y_{1}(t)}{d t}>y_{1}(t)\left[r_{1}-a_{1} y_{1}-\frac{b_{1} \varepsilon_{1}}{1+\varepsilon_{1}}\right]
$$

Applying Lemma 2.2 to (3.17), it follows that

$$
\lim _{t \rightarrow+\infty} y_{1}(t) \geq \frac{r_{1}-\frac{b_{1} \varepsilon_{1}}{1+\varepsilon_{1}}}{a_{1}} .
$$

Setting $\varepsilon \rightarrow 0$ leads to

$$
\lim _{t \rightarrow+\infty} y_{1}(t) \geq \frac{r_{1}}{a_{1}}
$$

This together with (3.3) shows that

$$
\lim _{t \rightarrow+\infty} y_{1}(t)=\frac{r_{1}}{a_{1}} .
$$

This completes the proof of Theorem 1.7 .

Proof of Theorem 1.9. Since the proof of Theorem 1.9 is similar to that of the proof of Theorem 1.7 , we omit the detail here.

\section{Numeric simulations}

Now let us consider the following examples.

\section{Example 4.1.}

$$
\begin{aligned}
& \frac{d y_{1}(t)}{d t}=y_{1}(t)\left[3-y_{1}-\frac{3 y_{2}}{1+y_{2}}\right] \\
& \frac{d y_{2}(t)}{d t}=y_{2}(t)\left[3-y_{2}-\frac{3 y_{1}}{1+y_{1}}\right] .
\end{aligned}
$$


Corresponding to system (1.1), one has

$$
r_{1}=r_{2}=b_{1}=b_{2}=3, a_{1}=a_{2}=1,
$$

and so,

$$
r_{1}\left(a_{2}+r_{2}\right)=12>9=b_{1} r_{2}, r_{2}\left(a_{1}+r_{1}\right)=12>9=b_{2} r_{1}
$$

and

$$
a_{2}-b_{2}+r_{2}=1 \neq 0
$$

Equations (4.2) and (4.3) show that all the conditions of Theorem 1.4 hold, and it follows from Theorem 1.4 that system (4.1) admits a unique positive equilibrium which is globally attractive. Fig. 1 also supports these findings. However, by simple computation, one can easily obtain that

$$
M_{1}=M_{2}=3
$$

and

$$
m_{1}=m_{2}=3-\frac{3 \times 3}{1+3}=\frac{3}{4}
$$

and so,

$$
a_{1}-\frac{b_{2}}{\left(1+m_{1}\right)^{2}}=1-\frac{3}{\left(1+\frac{3}{4}\right)^{2}}=-\frac{1}{49}<0, a_{2}-\frac{b_{1}}{\left(1+m_{2}\right)^{2}}=-\frac{1}{49}<0 .
$$

Which means that Theorem $\mathrm{C}$ of [23] could not be applied to system (4.1), and one can only obtain the persistent property of system (4.1) from [23]. Obviously, we improve the main results of [23] by deleting unnecessary conditions.

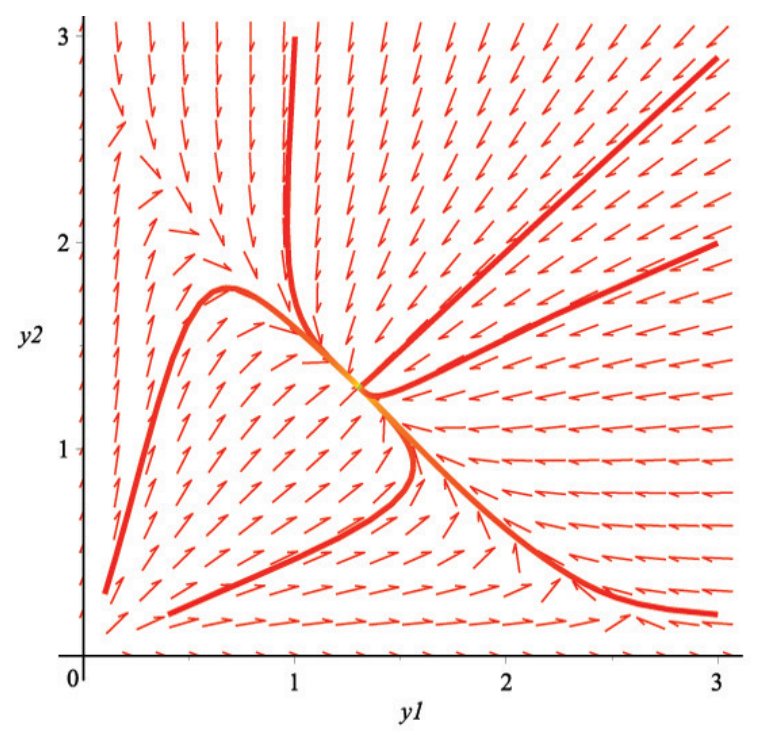

Figure 1: Dynamic behavior of system 4.1] with the initial condition $\left(y_{1}(0), y_{2}(0)\right)=(0.4,0.2),(1,3),(3,2.9),(3,0.2)$, $(3,2)$ and $(0.1,3)$, respectively. 


\section{Example 4.2.}

$$
\begin{aligned}
& \frac{d y_{1}(t)}{d t}=y_{1}(t)\left[3-y_{1}-\frac{3 y_{2}}{1+y_{2}}\right] \\
& \frac{d y_{2}(t)}{d t}=y_{2}(t)\left[3-y_{2}-\frac{8 y_{1}}{1+y_{1}}\right] .
\end{aligned}
$$

Corresponding to system (1.1), one has

$$
r_{1}=r_{2}=b_{1}=3, a_{1}=a_{2}=1, b_{2}=8
$$

and so,

$$
r_{1}\left(a_{2}+r_{2}\right)=12>9=b_{1} r_{2},
$$

and

$$
\left(a_{1}-b_{1}+r_{1}\right) r_{2}^{2}+\left(a_{1} a_{2}+a_{2} r_{1}+b_{1} b_{2}-b_{2} r_{1}\right) r_{2}-a_{2} b_{2} r_{1}=-3<0 .
$$

Equations (4.5) and (4.6) show that all the conditions of (1.11) hold, it follows from Theorem 1.7 that

$$
\lim _{t \rightarrow+\infty} y_{1}(t)=3, \lim _{t \rightarrow+\infty} y_{2}(t)=0 .
$$

Fig. 2 also supports these findings.

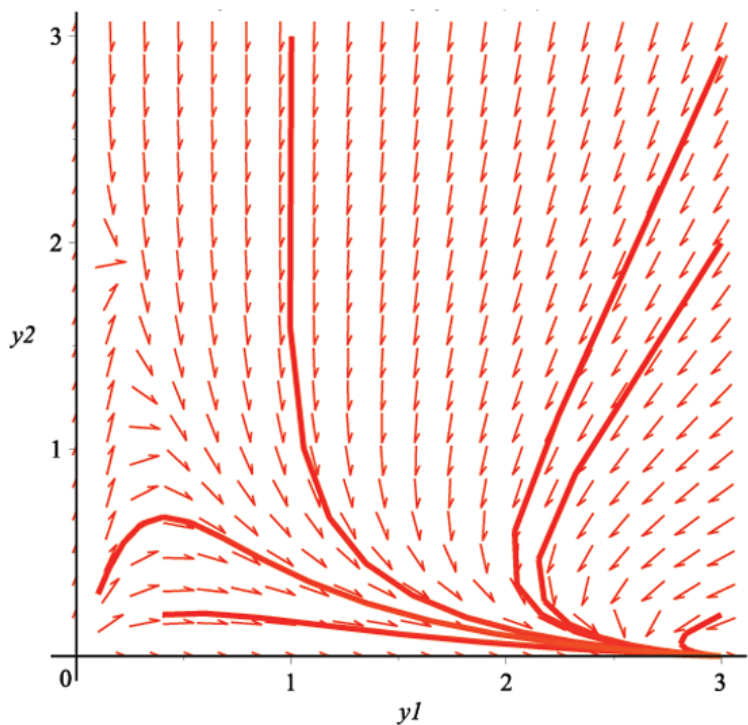

Figure 2: Dynamic behavior of system (4.4) with the initial condition $\left(y_{1}(0), y_{2}(0)\right)=(0.4,0.2),(1,3),(3,2.9),(3,0.2)$, $(3,2)$ and $(0.1,3)$, respectively.

\section{Discussion}

In this paper, we revisit the dynamic behaviors of a two-species competitive system with nonlinear inter-inhibition terms, which was proposed by Wang et al. [23]. By using the iterative method, we 
are able to obtain some more deep results. More precisely, we show that for the autonomous case of system (1.2), conditions which ensure the permanence of the system are almost enough to ensure the global stability of the system. We also investigate the extinction property of the system (1.1). Our results indicate that if the growth rate of the species is small enough and the rate of the intraspecific is large enough, then the species will be driven to extinction.

We mention here that a suitable population model should incorporate some past state of the species, and this will lead to a system with delay. Whether the delay has positive or negative influence on the dynamic behaviors of the system is still unknown, we leave this for future investigation.

\section{Acknowledgment}

This work is supported by National Social Science Foundation of China (16BKS132), Humanities and Social Science Research Project of Ministry of Education Fund(15YJA710002) and the Natural Science Foundation of Fujian Province (2015J01283).

\section{References}

[1] F. D. Chen, On a nonlinear nonautonomous predator-prey model with diffusion and distributed delay, J. Comput. Appl. Math., 180 (2005), 33-49. 1. 2

[2] F. D. Chen, Average conditions for permanence and extinction in nonautonomous Gilpin-Ayala competition model, Nonlinear Anal. Real World Appl., 7 (2006), 895-915. 1

[3] F. D. Chen, Some new results on the permanence and extinction of nonautonomous Gilpin-Ayala type competition model with delays, Nonlinear Anal. Real World Appl., 7 (2006), 1205-1222.

[4] L. J. Chen, J. T. Sun, F. D. Chen, L. Zhao, Extinction in a Lotka-Volterra competitive system with impulse and the effect of toxic substances, Appl. Math. Model., 40 (2016), 2015-2024.

[5] F. D. Chen, H. N. Wang, Dynamic behaviors of a Lotka-Volterra competitive system with infinite delays and single feedback control, J. Nonlinear Funct. Anal., 2016 (2016), 21 pages. 1

[6] F. D. Chen, H. N. Wang, Y. H. Lin, W. L. Chen, Global stability of a stage-structured predator-prey system, Appl. Math. Comput., 223 (2013), 45-53. 1

[7] F. D. Chen, X. D. Xie, Z. Li, Partial survival and extinction of a delayed predator-prey model with stage structure, Appl. Math. Comput., 219 (2012), 4157-4162. 1

[8] F. D. Chen, X. D. Xie, Z. S. Miao, L. Q. Pu, Extinction in two species nonautonomous nonlinear competitive system, Appl. Math. Comput., 274 (2016), 119-124. 1

[9] F. D. Chen, X. D. Xie, H. N. Wang, Global stability in a competition model of plankton allelopathy with infinite delay, J. Syst. Sci. Complex., 28 (2015), 1070-1079. 1

[10] F. D. Chen, M. S. You, Permanence for an integrodifferential model of mutualism, Appl. Math. Comput., 186 (2007), 30-34. 1

[11] K. Gopalsamy, Stability and oscillations in delay differential equations of population dynamics, Mathematics and its Applications, Kluwer Academic Publishers Group, Dordrecht, (1992). 1

[12] M. X. He, Z. Li, F. D. Chen, Permanence, extinction and global attractivity of the periodic Gilpin-Ayala competition system with impulses, Nonlinear Anal. Real World Appl., 11 (2010), 1537-1551. 1

[13] Z. Li, F. D. Chen, Extinction in periodic competitive stage-structured Lotka-Volterra model with the effects of toxic substances, J. Comput. Appl. Math., 231 (2009), 143-153. 1

[14] Z. Li, F. D. Chen, M. X. He, Asymptotic behavior of the reaction-diffusion model of plankton allelopathy with nonlocal delays, Nonlinear Anal. Real World Appl., 12 (2011), 1748-1758. 1

[15] Z. Li, F. D. Chen, M. X. He, Global stability of a delay differential equations model of plankton allelopathy, Appl. Math. Comput., 218 (2012), 7155-7163.

[16] Z. Li, M. Han, F. D. Chen, Global stability of a stage-structured predator-prey model with modified Leslie-Gower and Holling-type II schemes, Int. J. Biomath., 5 (2012), 13 pages.

[17] Y. H. Lin, X. D. Xie, F. D. Chen, T. T. Li, Convergences of a stage-structured predator-prey model with modified Leslie-Gower and Holling-type II schemes, Adv. Difference Equ., 2016 (2016), 19 pages. 1

[18] L. Q. Pu, X. D. Xie, F. D. Chen, Z. S. Miao, Extinction in two-species nonlinear discrete competitive system, Discrete Dyn. Nat. Soc., 2016 (2016), 10 pages. 1 
[19] W. J. Qin, Z. J. Liu, Y. P. Chen, Permanence and global stability of positive periodic solutions of a discrete competitive system, Discrete Dyn. Nat. Soc., 2009 (2009), 13 pages. 1

[20] C. L. Shi, Z. Li, F. D. Chen, Extinction in a nonautonomous Lotka-Volterra competitive system with infinite delay and feedback controls, Nonlinear Anal. Real World Appl., 13 (2012), 2214-2226. 1

[21] Q. L. Wang, Z. J. Liu, Uniformly asymptotic stability of positive almost periodic solutions for a discrete competitive system, J. Appl. Math., 2013 (2013), 9 pages. 1

[22] Q. L. Wang, Z. J. Liu, Z. X. Li, Positive almost periodic solutions for a discrete competitive system subject to feedback controls, J. Appl. Math., 2013 (2013), 14 pages.

[23] Q. L. Wang, Z. J. Liu, Z. X. Li, R. A. Cheke, Existence and global asymptotic stability of positive almost periodic solutions of a two-species competitive system, Int. J. Biomath., 7 (2014), 18 pages. 1, 1, 1, 4.1. 5

[24] X. D. Xie, F. D. Chen, Y. L. Xue, Note on the stability property of a cooperative system incorporating harvesting, Discrete Dyn. Nat. Soc., 2014 (2014), 5 pages. 1

[25] X. D. Xie, F. D. Chen, K. Yang, Y. L. Xue, Global attractivity of an integrodifferential model of mutualism, Abstr. Appl. Anal., 2014 (2014), 6 pages.

[26] K. Yang, X. D. Xie, F. D. Chen, Global stability of a discrete mutualism model, Abstr. Appl. Anal., 2014 (2014), 7 pages.

[27] S. B. Yu, Global asymptotic stability of a predator-prey model with modified Leslie-Gower and Hollingtype II schemes, Discrete Dyn. Nat. Soc., 2012 (2012), 8 pages. 1

[28] S. B. Yu, Permanence for a discrete competitive system with feedback controls, Commun. Math. Biol. Neurosci., 2015 (2015), 11 pages. 1

[29] Q. Yue, Dynamics of a modified LeslieGower predatorprey model with Holling-type II schemes and a prey refuge, SpringerPlus, 5 (2016), 1-12. 1 